\title{
CAMBIOS EN LAS PROPIEDADES QUÍMICAS DEL SUELO CON LA UTILIZACIÓN DE Dichotomius satanas (Harold, 1867) (COLEOPTERA: SCARABAEIDAE: SCARABAEINAE) EN CONDICIONES DE INVERNADERO
}

\author{
CHANGES IN SOIL CHEMICAL PROPERTIES USING Dichotomius satanas (Harold, 1867) (COLEOPTERA: \\ SCARABAEIDAE: SCARABAEINAE) IN GREENHOUSE CONDITIONS
}

\author{
Mercedes Girón-Vanderhuck, Janneth Molina-Rico, Oscar Alexander Aguirre-Obando \\ Docentes Programa Lic. Biología y Educación Ambiental, Universidad del Quindío, Grupo de Investigación en Biología Educativa BIOEDUQ.
}

Fecha de recibido: Febrero 3 de 2010

Fecha de aceptado: Junio 9 de 2010

Correspondencia: Programa Licenciatura en Biología y Educación Ambiental, Universidad del Quindío, Av. Bolivar calle 12 norte. Correo electrónico: mercedesgiron@uniquindio.edu.co

\section{RESUMEN}

El objetivo de este trabajo fue evaluar la actividad de los escarabajos coprófagos Dichotomius satanas Harold 1867, alimentados con excremento bovino y porcino, y su efecto sobre los nutrientes de un suelo empobrecido. Para esto, se colectaron ejemplares de D. satanas con 432 trampas pitfall distribuidas en tres sitios del Departamento del Quindío. Para el análisis de los datos se utilizó un diseño factorial de bloques completamente al azar con el fin de evaluar los efectos: escarabajos (presencia o ausencia) y tipo de excremento (bovino y porcino) repetidos 4 veces. Los suelos tratados sólo con excremento bovino y porcino experimentaron un descenso significativo en el $\mathrm{pH}$, la C.I.C y el contenido de $\mathrm{P}, \mathrm{K}, \mathrm{Ca}, \mathrm{Mg}, \mathrm{Zn}$ y $\mathrm{Mn}$. Por el contrario, los suelos tratados con $\mathrm{D}$. satanas y excremento bovino y porcino sufrieron un incremento significativo en el $\mathrm{pH}$, la materia orgánica, la C.I.C y el contenido de $\mathrm{P}, \mathrm{K}, \mathrm{Ca}, \mathrm{Mg}, \mathrm{Cu}, \mathrm{Zn}$ y $\mathrm{Mn}$; mientras que, se observó una disminución significativa en la concentración de Fe. El P fue uno de los elementos que más se incrementó (6 a 120 ppm) con la adición de excremento porcino y la actividad de los escarabajos. En este estudio se demuestra que la actividad de $D$. satanas aumenta significativamente la disponibilidad de nutrientes en un suelo empobrecido tipo andisol, lo que genera un cambio representativo en las propiedades químicas del suelo en tan sólo 45 días.

Palabras clave: Escarabajos coprófagos, nutrientes, excremento

\begin{abstract}
The aim of this study was to evaluate the activity of dung beetles Dichotomius satanas Harold 1867, fed on cattle and pig excrement, and its effect on soil nutrients of depleted soil nutrients. For this, we collected specimens of $D$. satanas with 432 pitfall traps on three sites at the Quindio Department. For data analysis we used a factorial design randomized complete block in order to evaluate the effects: beetles (presence or absence) and type of dung (cattle and pigs), repeated 4 times. The soils treated with cattle and pig excrement showed a significant decrease in $\mathrm{pH}$, C.I.C and the content of $P, K, C a, M g, Z n$ and $M n$. Whereas, soils treated with D. satanas and cattle and pig excrement were significant increases in $\mathrm{pH}$, organic matter, .C.I.C and the content of $\mathrm{P}, \mathrm{K}, \mathrm{Ca}, \mathrm{Mg}, \mathrm{Cu}, \mathrm{Zn}$ and $\mathrm{Mn}$; addition to this, there was a significant decrease in the concentration of Fe. The $P$ was one of the highest improvement (6 to $120 \mathrm{ppm}$ ) with the increasing of pig manure and beetles activity. This study showed that the activity of $D$. satanas significantly grows up the availability of nutrients in soil type andisol deteriorated, leading to a change in representative soil chemical properties in just 45 days.
\end{abstract}

Keywords: Dung beetles, nutrients, dung. 


\section{INTRODUCCIÓN}

En el departamento del Quindío se han reemplazado grandes coberturas boscosas por cultivos de café, y en la actualidad, debido a la disminución de los precios del café, se han eliminado gran parte de estos cultivos para implementar pastizales de explotación ganadera (Corporación Autónoma Regional de Quindío, 2004). Como consecuencia se ocasiona un severo deterioro ambiental, que afecta la estabilidad de los suelos y la cobertura vegetal existente. Además, la implementación de zonas de pastoreo implica una alta producción de excremento, que no sólo genera problemas sanitarios sino que afecta el crecimiento de las especies vegetales que sirven de alimento al ganado.

Generalmente el excremento del ganado bovino y porcino es considerado un elemento secundario de desecho dentro del proceso de explotación agropecuaria. Sin embargo, lejos de ser el punto final, es un eslabón más en la cadena productiva, ya que el excremento cuando se incorpora al suelo y es degradado, sus componentes quedan disponibles para las plantas (Fincher et al., 1981).

Existen insectos como los escarabajos coprófagos que utilizan el excremento de vertebrados como sustrato para la ovoposición y como fuente de alimento para adultos y larvas (Gill, 1991). Para ello, entierran el excremento en el suelo y, como consecuencia de esta actividad biológica, intervienen en el ciclado de nutrientes y en los procesos de conservación de la estructura edáfica (Bornemissza y Williams, 1970; McKinney y Morley, 1975; Fincher et al, 1981). Por consiguiente, ayudan a incrementar la concentración de nutrientes en el suelo y contribuyen a la sostenibilidad de los sistemas agrícolas y pastoriles (Omaliko, 1984; Brussard y Hijdra, 1986; Edwards y Aschenborn, 1987; Baethgen, 1992; Mittal, 1993; Herrick y Lal, 1995; Mariategui et al, 2001; Bertone, 2004; Lastro, 2006). Además, los escarabajos evitan la contaminación de los potreros, ya que, si el excremento bovino permanece en la superficie del suelo puede destruir las plantas o retardar el crecimiento, y hacer que crezcan plantas forrajeras de mal sabor (Mariategui et al, 2001).

A nivel mundial se ha reconocido el aporte de los escarabajos de la subfamilia Scarabaeinae en el ciclado de nutrientes edáfico. Bomemissza y Williams (1970), McKinney y Morley (1975), Fincher et al, (1981) y más recientemente Bertone (2004) confirmaron los efectos benéficos de estos escarabajos en los ecosistemas de pradera. Estos estudios, realizados en diferentes tipos de vegetación, indican un incremento en la toma de nutrientes por las plantas, optimizando así el valor nutricional para el ganado. Martínez y Lumaret (2006) hallaron un incremento en la producción forrajera de pastizales como consecuencia del reciclaje de materia orgánica, especialmente excremento. Sin embargo, observaron que al incrementar la ganadería, se aumentaba la cantidad de excremento depositado en el suelo, produciéndose una pérdida de los pastos. Horgan (2001) y Bertone (2004) afirman que el manejo de los pastizales y del ganado está estrechamente relacionado con la biología de los escarabajos coprófagos, en particular, con la remoción y descomposición del excremento.

En Colombia los escarabajos coprófagos han sido estudiados desde un punto de vista taxonómico y ecológico, principalmente a nivel de estructura de comunidades (Escobar, 1994; Amezquita, 1996; Martin-Piera y Fernadez, 1996; Medina y Kattan, 1996; Escobar, 1997; Pardo, 1997; Escobar, 2000; Escobar, 2004; Escobar et al., 2007; Amezquita et al., 1999; Medina et al., 2001; Medina et al., 2002; Molina, 2007), pero, no existe aporte alguno que resalte la función de éstos escarabajos en el ecosistema. De tal forma que su efecto en el ciclado de nutrientes no ha sido documentado para Colombia.

Por lo anterior, se buscó una alternativa para minimizar la pérdida de nutrientes edáficos y el impacto del excremento sobre el medio ambiente, a través de la actividad alimentaria de los escarabajos coprófagos. Es así como, el objetivo de este trabajo fue evaluar si la actividad de los escarabajos coprófagos Dichotomius satanas Harold 1867, alimentados con excremento bovino y porcino, tenía un efecto sobre los nutrientes de un suelo empobrecido. Esto con el propósito de optimizar un recurso que, bajo condiciones tropicales, pueda generar una dinámica eficiente en la recuperación de suelos. Lo que significaría un reto en la competitividad y sostenibilidad del sector agropecuario. 


\section{MATERIALES Y MÉTODOS}

Los individuos de D. satanas, se colectaron en septiembre de 2005 en el sendero ecológico Cedro Rosado de la Universidad del Quindío y en los bosques Bremen y Barbas ubicados en el municipio de Filandia, Quindío, así como en los pastizales adyacentes a estos bosques.

El sendero Cedro Rosado es un remanente de bosque secundario ubicado en el campus de Universidad del Quindío, al noroeste de la ciudad de Armenia, Departamento del Quindío ( $4^{\circ} 45^{\prime} \mathrm{N}, 75^{\circ} 45^{\prime} \mathrm{O}$ ), a una altitud de $1450 \mathrm{~m}$. Este fragmento de bosque posee una extensión de 8 ha y corresponde a la zona de vida bosque húmedo premontano (bh-PM). Los bosques Bremen y Barbas están ubicados en el municipio de Filandia, en el Departamento de Quindío a $4^{\circ} 40^{\prime} 6^{\prime \prime} \mathrm{N}$, $75^{\circ} 37^{\prime} 16^{\prime \prime} \mathrm{O}$. Presentan una transición de bosque muy húmedo premontano a montano bajo. Se encuentran a una altitud de $1812 \mathrm{~m}$. El primero cuenta con una extensión de 375,4 ha en bosque nativo y 365 ha en coníferas y, el segundo tiene una extensión de 2500 ha (C.R.Q, 2004). Los pastizales adyacentes a los bosques son de uso semi-intensivo de explotación ganadera, poseen manejo rotacional y están separados con cercas eléctricas.

Los ejemplares de D. satanas se capturaron con trampas de caída con excremento humano como cebo (Escobar, 1997), para esto se montaron 6 transectos por sitio: 3 en el fragmento boscoso y 3 en el potrero, ya que esta especie ocupa hábitats de transición. Cada transecto tenía una longitud de 660 m, 330 m en bosque y $330 \mathrm{~m}$ en potrero. Se colocaron 24 trampas de caída por transecto, separadas entre sí $25 \mathrm{~m}$. A las 24 y 48 horas de montadas las trampas, se colectaron todos los individuos de D. satanas y se transportaron vivos al insectario de la Universidad del Quindío, para establecer una colonia de mantenimiento. Algunos ejemplares se dejaron como referencia en el Museo de Artrópodos de la Universidad del Quindío (M.A.U.Q), bajo los códigos No 995 al 1000 del M.A.U.Q.

Se diseñó un arreglo factorial de bloques completamente al azar para evaluar los siguientes efectos: escarabajos (presencia o ausencia) y tipo de excremento (bovino y porcino) con 4 repeticiones. En el experimento se utilizaron 16 recipientes plásticos de $30 \mathrm{~cm} \times 20 \mathrm{~cm} \times 15 \mathrm{~cm}$, cada uno con dos $\mathrm{kg}$ de suelo empobrecido, obtenido de una pastura de la Finca Bengala de la Universidad del Quindío.

Para la evaluación de cada uno de los factores de análisis se procedió de la siguiente forma: los 16 recipientes se separaron en dos grupos, cada uno de ocho. Al primer grupo, se le agregó $10 \mathrm{~g}$ de D. satanas por recipiente (aproximadamente entre 9 a 11 individuos, machos y hembras), el otro, se dejo sin escarabajos. A su vez, cada grupo se separo en dos, cada uno conformado por cuatro recipientes, y se agregó, por recipiente, $40 \mathrm{~g}$ de excremento bovino a uno y $40 \mathrm{~g}$ de excremento porcino a otro. Este procedimiento se realizó cada 8 días, por un periodo de 45 días. Los recipientes se cubrieron con una malla de $0,2 \mathrm{~mm}$ para evitar que los escarabajos se escaparan. El excremento porcino se tomó de los cerdos de cría de la finca la Aldana de la Universidad del Quindío, alimentados con ITAL-CERDA CRÍA, y el excremento bovino se recogió en las instalaciones del Sena Agropecuario, donde los animales eran alimentados con pasto kingrass.

Al inicio del experimento, se tomaron 4 muestras de suelo (suelo control sin escarabajos y sin excremento) $y$, al final, se obtuvieron 4 muestras por cada tratamiento, y se analizaron las siguientes variables: fósforo (P), potasio (K), Calcio (Ca), Magnesio (Mg), cobre (Cu), Zinc (Zn), Manganeso (Mn) y Hierro (Fe), pH y materia orgánica (M.O) (Tabla 1). Los análisis se realizaron en el Laboratorio Químico de Suelos de la Universidad del Quindío. El nitrógeno no se evaluó debido a la volatilidad de este elemento en el suelo.

Tabla 1: Características del suelo obtenido en una pastura de la Granja Bengala ubicada en el Municipio de Filandia, Departamento del Quindío (2005).

\begin{tabular}{lc}
\hline Propiedad del suelo & Promedio \pm error estándar \\
\hline Fósforo (ppm) & $6,000 \pm 0,408$ \\
Potasio (meq $\left.100^{-1}\right)$ & $0,040 \pm 0,004$ \\
Calcio (meq $\left.100^{-1}\right)$ & $0,500 \pm 0,041$ \\
Magnesio (meq $\left.100^{-1}\right)$ & $0,100 \pm 0,011$ \\
Cobre (ppm) & $3,000 \pm 0,408$ \\
Zinc (ppm) & $3,000 \pm 0,408$ \\
Manganeso (ppm) & $5,000 \pm 0,406$ \\
Hierro (ppm) & $94,000 \pm 0,410$ \\
pH & $5,400 \pm 0,041$ \\
Materia orgánica $(\%)$ & $6,900 \pm 0,041$ \\
Textura & Franco arenoso \\
\hline
\end{tabular}


A las variables de $\mathrm{P}, \mathrm{K}, \mathrm{Ca}, \mathrm{Mg}, \mathrm{Cu}, \mathrm{Zn}, \mathrm{Mn}, \mathrm{Fe}, \mathrm{Ph}$ y materia orgánica se les comprobó la distribución normal de los datos y la homogeneidad de varianzas antes de proceder con el análisis de varianza multifactorial $2 \times 2 \times 4$, con el fin de evaluar las diferencias para los factores: escarabajos (presencia o ausencia) y excremento (bovino y porcino) con cuatro repeticiones. Cuando las diferencias fueron significativas se utilizó la prueba de Tukey para explicar las diferencias entre niveles de cada factor. Se utilizó la prueba de Dunnet para comparar los tratamientos con los controles.

Con el fin de identificar las variables que explican la mayor variabilidad de los nutrientes en el suelo producto de la actividad alimentaría de los escarabajos coprófagos, se realizó un análisis multivariado mediante la técnica de componentes principales, que permite encontrar las nuevas variables denominadas factores, que son una combinación lineal de todas las variables originales ( $\mathrm{P}, \mathrm{K}, \mathrm{Ca}, \mathrm{Mg}, \mathrm{Cu}, \mathrm{Zn}, \mathrm{Mn}, \mathrm{Fe}, \mathrm{pH}, \mathrm{M}$. O.) y pretende disminuir la cantidad de variables maximizando la varianza (Manly, 1991). Todos los análisis estadísticos se realizaron con el programa STATISTICA 7 (Statsoft Inc. 1984-2004).

\section{RESULTADOS}

La actividad de D. satanas en el suelo empobrecido generó diferencias significativas en las concentraciones de $\mathrm{P}, \mathrm{K}, \mathrm{Ca}, \mathrm{Mg}, \mathrm{Cu}, \mathrm{Zn}, \mathrm{Mn}, \mathrm{Fe}, \mathrm{Ph}$ y M.O. (Figura 1). Al comparar el suelo tratado con excremento y escarabajos con el suelo control (sin escarabajos y sin excremento) se evidenciaron diferencias significativas en la concentración de todas las variables de estudio $(P<0,01)$ (Tabla 2$)$, aunque este incremento se presentó de forma diferencial en cada uno de los nutrientes y tratamientos evaluados.

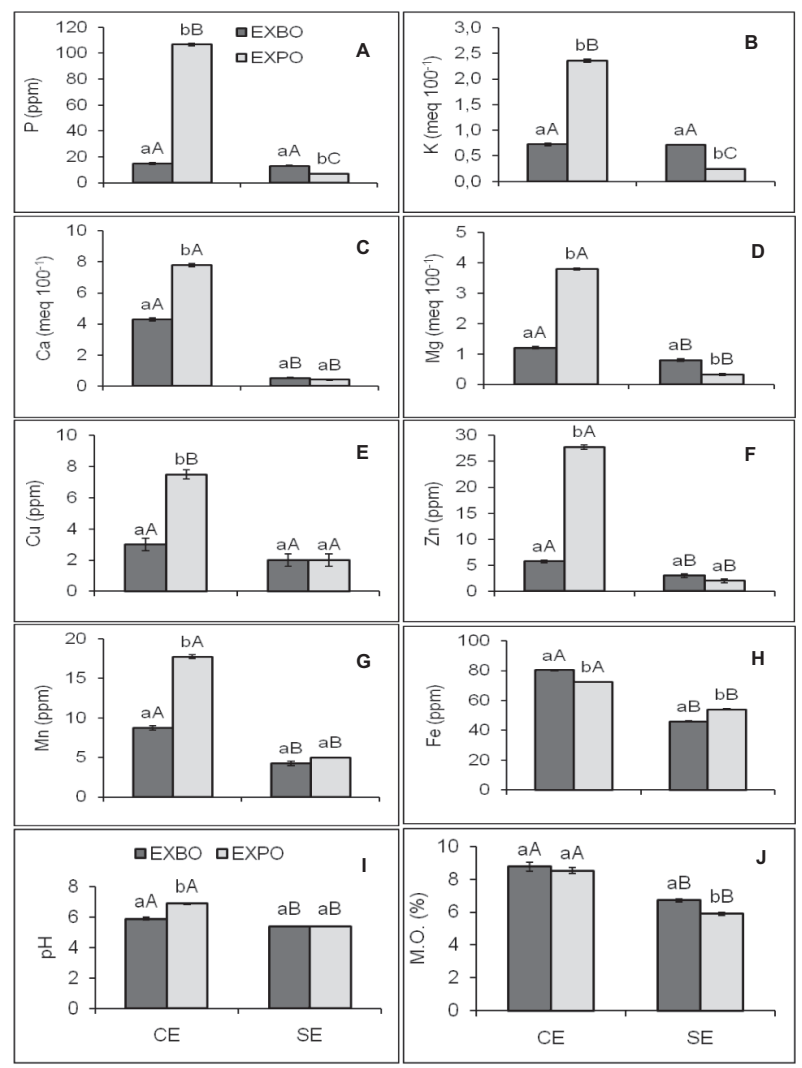

Figura 1. Valores medios de los macro y micronutrientes analizados en un suelo empobrecido sometido a los siguientes tratamientos: Con escarabajos y excremento bovino (CEXB), con escarabajos y excremento porcino (CEXP), sin escarabajos y con excremento bovino (SEXB), sin escarabajos y con excremento porcino (SEXP), (promedio \pm error estándar; $n=4)$. Los promedios seguidos con distintas letras dentro de cada tratamiento son significativamente distintos al 5\%. Las minúsculas comparan el efecto tratamiento (excremento y escarabajos). 
Tabla 2: Resultados del análisis de varianza para los diferentes parámetros edáficos, de acuerdo al factor escarabajo (con y sin) y factor excremento (bovino y porcino); ES = Escarabajo; $E X$ = Excremento. Los valores de $p$ que aparecen con asterisco presentan diferencias significativas $(p<0,05)$.

\begin{tabular}{|c|c|c|c|c|c|c|c|c|}
\hline \multirow{2}{*}{$\begin{array}{l}\text { Factor } \\
\text { analizado }\end{array}$} & \multicolumn{2}{|l|}{$\mathrm{pH}$} & \multicolumn{2}{|l|}{ M.O } & \multicolumn{2}{|l|}{$P$ (ppm) } & \multicolumn{2}{|c|}{$\mathrm{K}\left(\right.$ meq $\left.100^{-1}\right)$} \\
\hline & $\mathbf{F}$ & $p$ & $\mathbf{F}$ & $P$ & $\mathbf{F}$ & $p$ & $\mathbf{F}$ & $P$ \\
\hline ES & 240,000 & $0,000 *$ & 170,028 & $0,000^{*}$ & 5460,960 & $0,000 *$ & 2627,089 & $0,000^{*}$ \\
\hline EX & 60,000 & $0,000 *$ & 8,990 & $0,011^{*}$ & 3878,540 & $0,000 *$ & 791,133 & $0,000 *$ \\
\hline ES $x$ EX & 60,000 & $0,000 *$ & 2,572 & 0,135 & 5040,031 & $0,000 *$ & 2565,711 & $0,000 *$ \\
\hline \multirow{2}{*}{$\begin{array}{l}\text { Factor } \\
\text { analizado }\end{array}$} & \multicolumn{2}{|c|}{$\mathrm{Ca}\left(\right.$ meq $\left.100^{-1}\right)$} & \multicolumn{2}{|c|}{$\mathrm{Mg}\left(\right.$ meq $\left.100^{-1}\right)$} & \multicolumn{2}{|l|}{$\mathrm{Cu}(\mathrm{ppm})$} & \multicolumn{2}{|l|}{ Zn (ppm) } \\
\hline & $F$ & $p$ & $\mathbf{F}$ & $P$ & $\mathbf{F}$ & $p$ & $\mathbf{F}$ & $P$ \\
\hline ES & 5792,481 & $0,000^{*}$ & 2441,886 & $0,000^{*}$ & 72,428 & $0,000^{*}$ & 1299,600 & $0,000 *$ \\
\hline EX & 531,766 & $0,000^{*}$ & 739,712 & $0,000 *$ & 34,714 & $0,000 *$ & 705,600 & $0,000 *$ \\
\hline \multirow[t]{2}{*}{ ES $x$ EX } & & $0,000 *$ & 1536,668 & $0,000 *$ & 34,714 & $0,000 *$ & 846,400 & $0,000 *$ \\
\hline & \multicolumn{8}{|l|}{599,586} \\
\hline Factor & \multicolumn{2}{|l|}{ Mn (ppm) } & \multicolumn{2}{|l|}{ Fe (ppm) } & & & & \\
\hline analizado & $\mathbf{F}$ & $p$ & $F$ & $P$ & & & & \\
\hline ES & 909,343 & $0,000 *$ & 4308,480 & $0,000 *$ & & & & \\
\hline EX & 278,486 & $0,000 *$ & 0,100 & 0,761 & & & & \\
\hline ES $x$ EX & 362,143 & $0,000 *$ & 384,100 & $0,000^{*}$ & & & & \\
\hline
\end{tabular}

El suelo tratado con escarabajos y excremento sufrió un incremento significativo $(P<0,001)$ en la concentración del $P$ con respecto al control $(6,00$ ppm), pero, cuando al suelo se le agregó excremento porcino, en ausencia de escarabajos, este no experimentó un cambio significativo $(P>0,05)$ en la concentración del P (Tabla 2). Además, se encontró interacción significativa entre los factores excremento y escarabajos $(F=5040,031 ; d f=12 ; P=0,000)($ Tabla
3), el efecto de la interacción se observa en el suelo con escarabajos, en el sentido de que el tratado con excremento bovino presentó menor concentración de $P$ que el tratado con excremento porcino, mientras que, el suelo sin escarabajos presentó mayor concentración de este elemento $(P<0,001)$ cuando se trató con excremento bovino que con excremento porcino (Fig. 1A). 
Tabla 3. Resultados de la prueba de medias de Dunnett para los diferentes parámetros del suelo empobrecido sometido a diferentes tratamientos: $\mathrm{SESX}=$ suelo sin escarabajos y sin excremento (control); $\mathrm{CEXB}=$ con escarabajos y excremento bovino; $\mathrm{CEXP}=$ con escarabajos y excremento porcino; $S E X B$ = Sin escarabajos con excremento bovino; $S E X P$ = sin escarabajos con excremento porcino. Los valores de $p$ que aparecen con asterisco presentan diferencias significativas $(p<0.05)$.

\begin{tabular}{|c|c|c|c|c|c|c|c|}
\hline \multirow[t]{2}{*}{ Control } & Tratamiento & $\mathrm{pH}$ & M.O (\%) & $\mathbf{P}(\mathrm{ppm})$ & $\begin{array}{l}\text { K } \\
\text { (meq } 100^{-} \\
\text {1) }\end{array}$ & $\begin{array}{l}\mathrm{Ca} \\
(\mathrm{meq} \\
\left.100^{-1}\right)\end{array}$ & $\begin{array}{l}\mathrm{Mg} \\
(\mathrm{meq} \\
\left.100^{-1}\right)\end{array}$ \\
\hline & & $\bar{P}$ & $P$ & $P$ & $p$ & $P$ & $p$ \\
\hline \multirow[t]{4}{*}{ SESX } & CEXB & $0,000^{*}$ & $0,000^{*}$ & $0,000^{*}$ & $0,000^{*}$ & $0,000^{*}$ & $0,000 *$ \\
\hline & CEXP & $0,000^{*}$ & $0,000 *$ & $0,000^{*}$ & $0,000^{*}$ & $0,000^{*}$ & $0,000 *$ \\
\hline & SEXB & 1,000 & 0,856 & $0,000^{*}$ & $0,000 *$ & 1,000 & $0,000 *$ \\
\hline & SEXP & 1,000 & $0,002 *$ & 0,649 & $0,000^{*}$ & 0,659 & $0,001^{*}$ \\
\hline \multirow[t]{2}{*}{ Control } & Tratamiento & $\begin{array}{l}\mathrm{Cu} \\
(\mathrm{ppm})\end{array}$ & $\begin{array}{l}\text { Zn } \\
(\mathrm{ppm})\end{array}$ & Mn (ppm) & $\begin{array}{l}\mathrm{Fe} \\
(\mathrm{ppm})\end{array}$ & & \\
\hline & & $\bar{p}$ & $P$ & $P$ & $p$ & & \\
\hline \multirow[t]{4}{*}{ SESX } & CEXB & 1,000 & $0,000^{*}$ & $0,001^{*}$ & $0,000^{*}$ & & \\
\hline & CEXP & $0,000^{*}$ & $0,000^{*}$ & $0,000^{*}$ & $0,000^{*}$ & & \\
\hline & SEXB & 0,247 & 1,000 & $0,015^{*}$ & $0,000^{*}$ & & \\
\hline & SEXP & 0,247 & 0,266 & $0,000^{*}$ & $0,000 *$ & & \\
\hline
\end{tabular}

El suelo que contenía escarabajos y excremento y el tratado sin escarabajos y con excremento experimentaron un incremento significativo $(P<$ 0,001 ) en la concentración del $\mathrm{K}$ y $\mathrm{Mg}$ con respecto al control (0,04 y 0,1 meq 100-1 respectivamente). Asimismo, se encontró interacción significativa para el $\mathrm{K}(\mathrm{F}=2565,711 ; \mathrm{df}=12 ; \mathrm{P}=0,000)$ y el $\mathrm{Mg}(\mathrm{F}=$ 1536,$668 ; \mathrm{df}=12 ; \mathrm{P}=0,000$ ) entre los factores excremento y escarabajos, el efecto de la interacción se observa en el suelo con escarabajos, en el sentido de que el suelo tratado con excremento porcino presentó mayor concentración de $\mathrm{K}$ y Mg $(P<0,001)$ que el tratado con excremento bovino, mientras que el suelo sin escarabajos experimentó mayor concentración de estos elementos cuando se trató con excremento bovino que con excremento porcino (Figs. 1B y 1D).

Cuando al suelo con escarabajos se le aplicó excremento, se observó un incremento significativo ( $\mathrm{P}$ $<0,001)$ en la concentración de $\mathrm{Ca}$, con respecto al control (0,50 meq 100-1), mientras que el suelo sin escarabajos y con excremento no experimentó cambio alguno (Tabla 2). Igualmente, los factores excremento y escarabajos mostraron interacción significativa $(F=$ 599,586; $d f=12 ; P=0,000$ ) (Tabla 3). Este efecto se observa en el suelo con escarabajos, en el sentido de que el tratado con excremento bovino presentó menor concentración de Ca que el tratado con excremento porcino, mientras que, el suelo sin escarabajos no mostró diferencias significativas $(P>0,05)$ cuando se agregó excremento (Fig. 1C).

El Cu incrementó significativamente $(P<0,001)$ en el suelo que contenía escarabajos y excremento porcino, con respecto al control (3,00 ppm), mientras que no se diferenció significativamente $(P>0,05)$ en el suelo tratado con escarabajos y excremento bovino y el tratado sólo con excremento, sin escarabajos (Tabla 2). Además, se encontró interacción significativa para el $\mathrm{Cu}(\mathrm{F}=34,714 ; \mathrm{df}=12 ; \mathrm{P}=0,000)$ entre los factores 
excremento y escarabajos, el efecto de la interacción se observa en el suelo con escarabajos, en el sentido de que el suelo tratado con excremento porcino presentó mayor concentración de $\mathrm{Cu}$ que el tratado con excremento bovino, mientras que este elemento no se diferenció significativamente $(P>0,05)$ en el suelo sin escarabajos tratado con los dos tipos de excremento (Tabla 3, Fig. 1E).

La concentración de $\mathrm{Zn}$, con respecto al control (3,00 ppm), incrementó significativamente $(P<0,001)$ cuando al suelo con escarabajos se le aplicó excremento porcino, mientras que con excremento bovino no experimentó cambio alguno (Tabla 2). Se encontró interacción significativa entre los factores excremento y escarabajos ( $F=846,400 ; d f=12 ; \mathrm{P}=$ 0,000 ) (Tabla 3), el efecto de la interacción se observa en que en el suelo tratado sólo con excremento, sin escarabajos, no se encontraron diferencias significativas $(P>0,05)$, mientras que el tratado con escarabajos y excremento porcino presentó mayor concentración de $\mathrm{Zn}$ que el tratado con excremento bovino (Tabla 3, Fig. 1F).

El $\mathrm{Mn}$ incrementó significativamente $(P<0,001)$ en el suelo que contenía escarabajos y excremento, con respecto al control (5,00 ppm), mientras que no se diferenció significativamente $(P>0,05)$ (Tabla 2 ) en el suelo tratado sólo con excremento, sin escarabajos. Además, se encontró interacción significativa para el $\mathrm{Mn}(\mathrm{F}=34,714 ; \mathrm{df}=12 ; \mathrm{P}=0,000)$ entre los factores excremento y escarabajos, el efecto de la interacción se observa en el suelo con escarabajos, en el sentido de que el suelo tratado con excremento porcino presentó mayor concentración de $\mathrm{Mn}$ que el tratado con excremento bovino, mientras que estos elementos no se diferenciaron significativamente $(P>0,05)$ en el suelo sin escarabajos tratado con los dos tipos de excremento (Tabla 3, Fig. 1G).

El suelo tratado con escarabajos y excremento y el tratado sin escarabajos con excremento experimentaron un descenso significativo $(P<0,001)$ en la concentración del Fe con respecto al control (94,00 ppm) (Tabla 2). Se encontró interacción significativa entre los factores excremento y escarabajos ( $F=384,100 ; \mathrm{df}=12 ; \mathrm{P}=0,000)$, el efecto de la interacción se observa en el suelo tratado con escarabajos, en el sentido de que el suelo tratado con excremento porcino presentó menor concentración de Fe que el tratado con excremento bovino, mientras que el suelo sin escarabajos tratado con excremento porcino presentó mayor concentración de Fe $(P<$ 0,001) que el tratado con excremento bovino (Tabla 3, Fig. $1 \mathrm{H})$.

El pH incrementó significativamente $(P<0,001)$ en el suelo tratado con escarabajos y excremento, con respecto al control $(5,40)$, mientras que no se diferenció significativamente $(P>0,05)$ en el suelo tratado sólo con excremento, sin escarabajos (Tabla 2). Además, se encontró interacción significativa entre los factores excremento y escarabajos (F: 60,00; df =12; P $=0,000$ ), el efecto de la interacción se observa en el suelo tratado con escarabajos y excremento, en el sentido de que el suelo tratado con excremento porcino presentó $\mathrm{pH}$ más alto que el tratado con excremento bovino, mientras que el $\mathrm{pH}$ del suelo sin escarabajos y excremento porcino no se diferenció significativamente del tratado con excremento bovino $(P>0,05)$ (Tabla 3, Fig. 1I).

La M.O no cambió significativamente $(P>0,05)$ con respecto al control (6,9 \%), cuando los suelos se trataron sólo con excremento bovino, pero, si experimentó un cambio significativo con excremento porcino y cuando se adicionaron escarabajos coprófagos y excremento (Tabla 2). No se encontró interacción significativa entre los factores escarabajos y excremento $(F=2,572 ; d f=12 ; P=0,135)$ (Tabla 3 y Fig. 1J).

En el análisis factorial de componentes principales, la absorción de varianzas permite identificar alguna tendencia de ordenación. La Figura 2 muestra la ordenación de los tratamientos en el plano factorial definido por los dos primeros factores del análisis de componentes principales. El factor 1 absorbe el $85,61 \%$ de la varianza total. En la parte positiva del factor se sitúa el suelo tratado sin escarabajos y excremento y en la parte negativa se dispone el suelo tratado con escarabajos y excremento, siendo los factores de carga más importantes el Mn $(-0,989), \mathrm{Ca}$ ($0,985), \mathrm{pH}(-0,983)$ y $\mathrm{Mg}(-0,976)$. Así pues, el primer factor refleja una separación entre el suelo tratado con escarabajos y el tratado sin escarabajos, al oponer los factores de carga de las variables analizadas. 


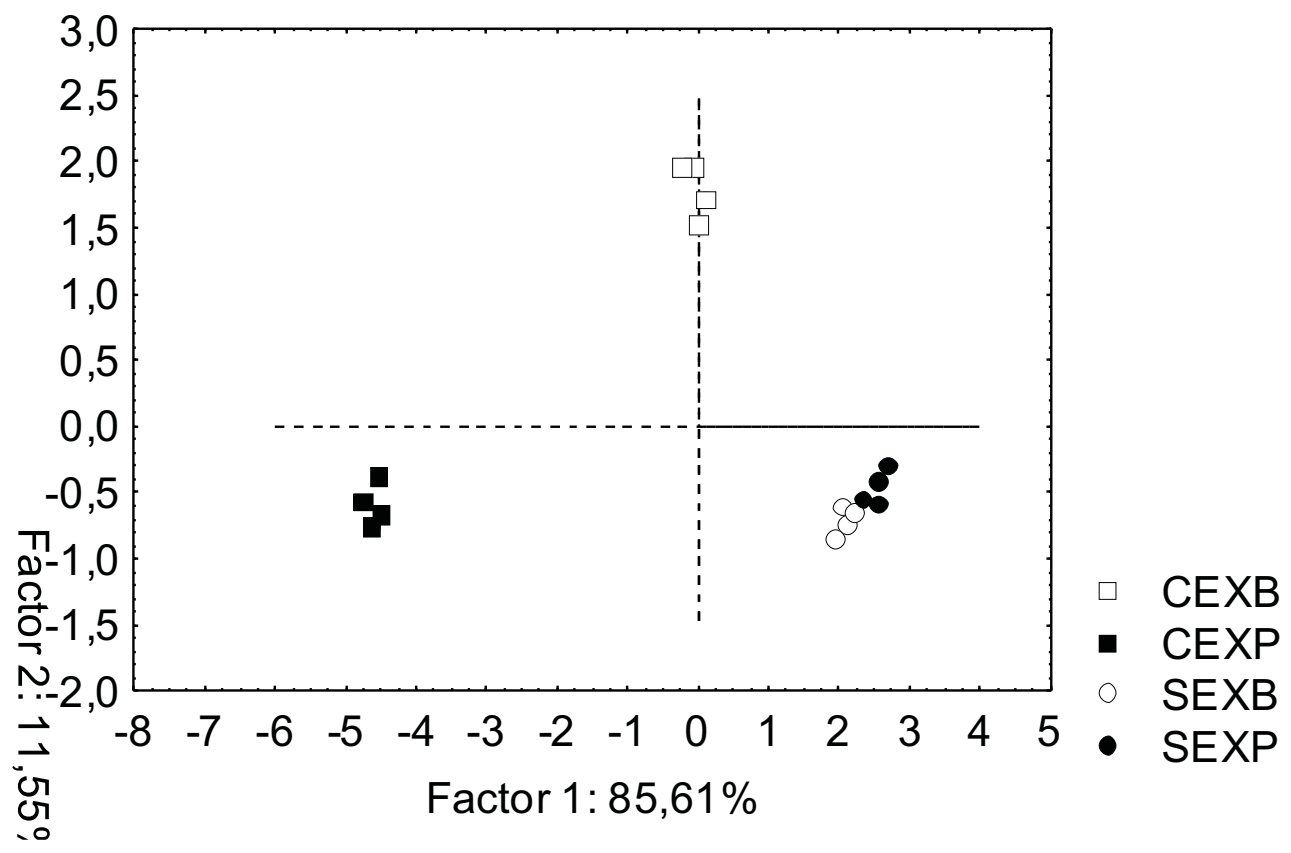

Figura 2. Ubicación de los diferentes tratamientos en el plano factorial definido por los dos primeros factores del análisis de componentes principales. Con escarabajos y excremento bovino (CEXB), con escarabajos y excremento porcino (CEXP), sin escarabajos y excremento bovino (SEXB), sin escarabajos y excremento porcino (SEXP).

El factor 2 absorbe el $11,55 \%$ de la varianza total. En la parte positiva del factor se sitúa el suelo tratado con escarabajos y excremento bovino. Este tiene como factores de carga más destacados el Fe $(0,713)$ y la M.O. (0,550). El suelo tratado con escarabajos y excremento porcino y el tratado sin escarabajos y con excremento se disponen en la parte negativa del factor 2 , y los factores de carga principales son el $P(-0,317)$, el $\mathrm{K}(-0,269)$ y el $\mathrm{Zn}(-0,243)$. Con respecto a este factor, existe una clara separación entre el suelo tratado con escarabajos y excremento bovino y los demás tratamientos.

\section{DISCUSIÓN}

El incremento en el $\mathrm{pH}$, la materia orgánica y el contenido de $\mathrm{P}, \mathrm{K}, \mathrm{Ca}, \mathrm{Mg}, \mathrm{Cu}, \mathrm{Zn}$ y $\mathrm{Mn}$ en el suelo tratado con D.satanas, también fue observado por Lastro (2006) en suelos tratados con Onthophagus taurus Schreber y excremento bovino. Asimismo, Bertone (2004) registró un incremento en el pH, la materia orgánica, la C.I.C. y la concentración de P, K, Ca, $\mathrm{Mg}, \mathrm{Zn}$ y $\mathrm{Cu}$, en suelos limo arenosos tratados con Onthophagus gazella Fabricius y excremento bovino. Igualmente, Rougon et al, (1988) hallaron un incremento en el $\mathrm{pH}$, la materia orgánica y las concentraciones de $\mathrm{K}, \mathrm{Ca}, \mathrm{Mg}$ en suelos tropicales ferruginosos tratados con escarabajos coprófagos y excremento de cebú. Por lo cual, puede sostenerse que la adición de excremento y su procesamiento, es decir, enterramiento y elaboración de túneles, por parte de D.satanas, contribuyeron a acelerar el retorno de los nutrientes en el suelo empobrecido. Esta acción, según varios autores, produce un incremento en la relación área/superficie del estiércol y una disminución en el contenido de humedad, lo que genera condiciones aeróbicas para el establecimiento de poblaciones de microorganismos, en su mayoría hongos, que ayudan a descomponer el sustrato orgánico y modificar las condiciones ambientales que afecta la liberación de nutrientes (Stevenson y Dindal, 1987; Yokohama et al., 1991; Bang et al., 2005).

El P fue uno de los elementos que más se incrementó con la adición de excremento porcino y la actividad de los escarabajos. Esto coincide con lo señalado por Vitosh et al., (1973), Omaliko (1984) y Singh et al., (1995) quienes han reportado incrementos en la disponibilidad de fósforo, que varían entre 1,7 y 2,7 veces, mediante la aplicación de excremento a lotes no enmendados. Yokoyama et al., (1991) explica que cuando el excremento es enterrado por los escarabajos, se produce un incremento en la actividad microbiana lo que trae consigo un aumento en la 
actividad de enzimas fosfatasas que liberan los fosfatos de compuestos orgánicos. Es decir, en el suelo se encuentran microorganismos capaces de producir altas cantidades de ácidos orgánicos (Osorio y Habte, 2001) y el crecimiento de estas poblaciones microbianas puede incrementarse con la actividad de los escarabajos coprófagos (Breymeyer et al., 1975). Según Bolan et al., (1994) el estiércol generalmente contiene ácidos orgánicos que son efectivos para solubilizar fosfatos inorgánicos. Hue (1991) lo comprobó adicionando ácidos orgánicos a tres Andisoles, un oxisol, un vertisol y un ultisol y, encontró un incremento en la disponibilidad de P.

La no evidencia de un cambio significativo en el pH, la materia orgánica y las concentraciones de $\mathrm{P}, \mathrm{Ca}, \mathrm{Cu}$ y $\mathrm{Zn}$, cuando el suelo fue tratado solo con excremento, puede, posiblemente, deberse a que se necesitan más de 45 días para que se descomponga el excremento y se liberen los nutrientes, ya que, según Osorio (2003) el incremento de nutrientes, generalmente, se produce a largo plazo, debido a que la descomposición del excremento es un proceso demorado. Este investigador encontró que suelos Andisoles ácidos, con alto contenido de $\mathrm{Fe}$ y $\mathrm{Al}$, mejoraban la disponibilidad de nutrientes y disminuían la cantidad de $\mathrm{Fe}$ y $\mathrm{Al}$, cuando se trataban con excremento porcino durante 10 años. Asimismo, Omaliko (1984) registró un incremento en las concentraciones de N, P, $\mathrm{Mg}$, y Ca a los 56 días de la deposición de excremento en el suelo. Otros autores (lyamuremye et al., 1996; Hue, 1992; Osorio et al., 2001) también han encontrado que la adición de excremento, aplicada a diferentes tipos de suelos, incrementa el $\mathrm{pH}$ y reduce la capacidad de fijación del fósforo, con un aumento en la disponibilidad de éste.

El análisis de componentes principales permitió visualizar el efecto de $D$. satanas sobre las variables analizadas. Con respecto al suelo tratado con escarabajos y excremento porcino, el incremento en casi todas las variables indica que probablemente la porcinaza es más rica en nutrientes que el excremento bovino y que $D$.satanas tiene preferencia por este tipo de excremento, más que por el bovino. De hecho, los escarabajos ayudan a acelerar el regreso de nutrientes al suelo (Stevenson y Dindal, 1987), mientras que el proceso es mucho más demorado cuando sólo se agrega excremento sin la presencia de los escarabajos (Osorio, 2003). Según Aarons et al. (2004), el mecanismo de entrada de nutrientes al suelo desde el excremento puede ser determinado por los cambios en la concentración de nutrientes mientras se está descomponiendo. Esta incorporación de nutrientes al suelo esta mediada en un alto porcentaje por la fauna del suelo, principalmente lombrices de tierra y escarabajos coprófagos. De esta forma, se demuestra la importancia de los escarabajos coprófagos en el ciclado de nutrientes en ecosistemas terrestres y se señala su uso potencial, para la recuperación de la fertilidad de los suelos en sistemas agrícolas.

\section{CONCLUSIONES}

La actividad de Dichotomius satanas incremento significativamente el $\mathrm{pH}$ y las concentraciones de $\mathrm{P}, \mathrm{K}$, $\mathrm{Ca}, \mathrm{Mg}, \mathrm{Cu}, \mathrm{Zn}$ y $\mathrm{Mn}$ del suelo empobrecido evaluado, cuando a este se le agregaba excremento porcino y bovino, pero, este aumento fue más notorio con la adición de excremento porcino. Mientras que, en el suelo sin escarabajos y con excremento, no se evidenciaron incrementos significativos en las variables estudiadas. Por consiguiente, D. satanas es más eficiente en la recuperación de suelos empobrecidos cuando se alimenta con excremento porcino que con bovino; además, los nutrientes del excremento retornan más rápidamente al suelo con la presencia de esta especie de escarabajo, que en su ausencia. Este hallazgo ratifica la importancia de D. satanas no sólo en la recuperación, a corto plazo, de suelos empobrecidos, sino que, además, la erige como una especie de valor económico en la producción agropecuaria, ya que puede utilizarse en la remoción de excremento porcino con la ganancia adicional de fertilizar el suelo en este proceso.

\section{AGRADECIMIENTOS}

Este trabajo fue posible gracias al apoyo financiero del la Vicerrectoría de Investigaciones de la Universidad del Quindío. Agradecemos a Nancy Forero Directora del Laboratorio de Suelos de la Universidad del Quindío por la asesoría prestada y al auxiliar de investigación John Alexander Sánchez, estudiante del programa de Biologia de la Universidad del Quindío. 


\section{BIBLIOGRAFÍA}

Amezquita, S. (1996). Los coleópteros coprófagos (Scarabaeidae: Scarabaeinae) en Colombia: Diversidad y distribución. Programa de inventarios de biodiversidad. Bogotá Instituto Alexander Von Humboldt. Informe $\mathrm{N}^{\circ} 10$.

Amezquita, S., Forsyth, A., Lopera, A. y Camacho, A. (1999). “Comparación de la composición y riqueza de especies de escarabajos coprófagos Coleoptera: Scarabaeinae en remanentes de bosque de la Orinoquía Colombiana". En Acta Zoologica Mexicana, (76), 113-126.

Aarons, S. R., Connor, C. R. y Gourley, C. J. (2004). “Dung decomposition in temperate dairy pastures. Change in soil chemical properties". En Australian Journal of Soil Research, (42), 107-114.

Baethgen, W. (1992). "Dinámica del nitrógeno en sistemas de rotación cultivos-pasturas". En: Morón, A y Baethgen, W. (Eds.). Sustentabilidad de las rotaciones cultivo-pastura en el Cono Sur; (3-25). Uruguay: Revista INIA Investigaciones Agropecuaria, (1).

Bang, H. S., Joon-ho, L., Kwon, O. S., Na, Y. E., Jang, Y. S. y Kim, W. H. (2005). "Effects of paracoprid dung beetles (Coleoptera : Scarabaeidae) on the growth of pasture herbage and on the underlying soil". En Applied Soil Ecology, (29), 165-171.

Bertone, M. A. (2004). Dung beteles (Coleoptera: Scarabaeidae and Geotrupidae) of North Carolina cattle pastures and their implications for pasture improvement. Thesis Master of Science. Faculty of North Carolina State University.

Bolan, N. S., Naidu, R., Mahimairaja, S. y Baskaran, S. (1994). "Influence of low-molecular-weight organic acids on the solubilization of phosphates". En Biology \& Fertility of Soils, (18), 311-319.

Bornemissza, G. F. y Williams, C. H. (1970). “An effect of dung beetle activity on plant yield”. En Pedobiología, 10, 1-7.

Breymeyer, A., Jakubezyk, H. y Olechowics, E. (1975). "Influence of coprophagous arthropods on microorganisms in sheep feces laboratory investigations". En Bulletin de L'academie Polonaise des Science, Serie des Science Biologiques, (23), 257-262.

Brussaard, L. y Hijdra, R. D. (1986). "Some effects of scarab beetles in sandy soils of the Netherlands". En Goederma, (37), 325-330.

Corporación Autónoma Regional Del Quindío. (2004). Plan de Gestión Ambiental para el Departamento del Quindío. 2004-2006. Consultada Mayo 10, 2005, en http//crq.gov.co/visual/plan_accion.05/html.

Edwards, P. B. y Aschenborn, H. H. (1987). "Patterns of nesting and dung beetles: implications for pasture productivity and fly control". En Journal of applied Ecology, (24), 837-852.

Escobar, F. (1994). Excrementos, coprófagos y deforestación en bosques de montaña del sur-occidente de Colombia. Tesis de pregrado Biología-Entomología. Universidad del Valle.

Escobar, F. (1997). "Estudio de la comunidad de coleópteros coprófagos (Scarabaeidae) en un remanente de bosque seco al norte del Tolima, Colombia”. En Caldasia, (19), 419-430.

Escobar, F. (2000). “Diversidad de escarabajos Coprófagos (Scarabaeidae: Scarabaeinae) en un Mosaico de Hábitats en la Reserva Natural Nukak, Guaviare.Colombia”. En Acta Zoologica Mexicana, (79), 103-121.

Escobar, F. (2004). "Diversity and composition of dung beetle (Scarabaeinae) assemblages in a heterogeneous Andean landscape". En Tropical Zoology, (17), 123-136. 
Escobar, F., Halffter, G. y Arellano, L. (2007). "From forest to pasture: an evaluation of the influence of environment and biogeography on the structure of dung beetle (Scarabaeinae) assemblages along three altitudinal gradients in the neotropical region". En Ecography, (30), 193-298.

Fincher, G. T., Monson, W. G. y Burton G. W. (1981). "Effects of cattle feces rapidly buried by dung beetles on yield and quality of coastal bermudagrass". En Agronomy Journal, (73), 775-779.

Gill, B.D. (1991). "Dung beetles in tropical american forests". En: Hanski, I. y Cambefort, Y. (Eds.). Dung Beetle Ecology. (211-230), Princeton, Princeton University Press.

Herrick, J. E. y Lal, R. (1995). "Soil physical property changes during dung decomposition in a tropical pasture". En Soil Science Society of America Journal, (59), 908-912.

Horgan, F.G. (2001). "Burial of bovine dung by coprophagous beteles (Coleoptera: Scarabaeidae) from horse and cow grazing sites in El Salvador". En European Journal of Soil Biology, (37), 103-111.

Hue, N. V. (1991). "Effects of organic acids/anions on P sorption and phytoavailability in soils with differrent mineralogies". En Soil Science, (152), 463-471.

Iyamuremye, F., Dick, R. P. y Baham, J. (1996). "Organic amendments and phosphorus dynamics: I. Phosphorus chemistry and sorption". En Soil Science, (161:7), 426-435.

Lastro, E. (2006). Dung beteles (Coleoptera: Scarabaediae and Geotrupidae) in North Carolina Pasture Ecosystem. Thesis Master of Science. Faculty of North Carolina State University. Consultado Junio 15, 2007, en http://www.lib.ncsu.edu/theses/available/etd-11022006-081712/unrestricted/etd.pdf

Mariategui, P., Speicys, C., Urretabizhaya, N. y Fernández, E. (2001). "Efecto de Ontherus sulcator F. (Coleoptera : Scarabaeidae) en la incorporación de estiércol al suelo". En Zootecnia Tropical, (19:2), 131-138.

Martin-Piera, F. y Fernandez, A. (1996). "Coleópteros de la Sierra de Chiribequete (Depto Caquetá, Colombia)". En Elytron, (10), 23-50.

Martinez, M. I. y Lumaret, J. P. (2006). "Las prácticas agropecuarias y sus consecuencias en la entomofauna y el entorno ambiental”. En Folia Entomológica Mexicana, (45:1), 57-68.

Mckinney, G. T. y Morley, F. H. (1975). “The agronomic role of introduced dung beetles in grazing systems”. En: Journal of Applied Ecology, (12), 831-837.

Manly, B. (1991). Multivariate Statistical Methods. London. Chapman \& Hall.

Medina, C. A. y Kattan, G. H. (1996). “Diversidad de coleópteros coprófagos (Scarabaeidae) de la reserva forestal de Escarlete". En Cespedesia, (21), 89-102.

Medina, C., Lopera-Toro, A., Vitolo, A. y Gill, B. (2001). "Escarabajos Coprófagos (Coleoptera: Scarabaeidae: Scarabaeinae) de Colombia". En Biota Colombiana, (2:2), 131-144.

Medina, C., Escobar. F. y Kattan, G. (2002). "Diversity and Habitat Use of Dung Beetles in a Restored Andean Landscape". En Biotropica, (34:1), 124-130.

Mittal, I. C. (1993). “Natural manuring and soil conditioning by dung beetles”. En Tropical Ecology, (34), 150-159.

Molina, L. J. (2007). Patrones espaciales de la comunidad de escarabajos coprófagos en paisajes modificados. Armenia: Elizcom. 
Omaliko, C. P. (1984). "Dung decomposition and its effects on the soil component of a tropical grassland ecosystem". En Tropical Ecology, (25), 214-220.

Osorio, N. W. y Habte, M. (2001). "Synergistic influence o fan arbuscular mycorrhizal fungus and a P solubilizing fungus on growth and P uptake of Leucaena leucocephala in a oxisol". En Arid Land Research and Management, (15), 263-274.

Osorio, N. W., Delisle, L. y Hue, N. V. (2001). Effect of animal manure on phosphate solubility and availabilitiy in three tropical soils. Department of Tropical Plant and Soil Sciences, University of Hawaii.

Osorio, N. W. (2003). Cambios en la fertilidad del suelo con la aplicación de materiales orgánicos. En: Memorias del Seminario Materiales Orgánicos en la Agricultura. Medellín. Sociedad Colombiana de la Ciencia del Suelo.

Pardo, L.C. 1997. "Muestreo preliminar de los escarabajos copronecrofílicos (Coleóptera - Scarabaeidae) de las selvas de la Fragua, cuenca baja río Calambre (Valle)". En Cespedesia, (22:69)

Rougon, D., Rougon, C., Trichet, J. y Levieux, J. (1998). “Enrichissement en matière d un sol sahélien au Niger par les Insectes coprophages (Coleoptera, Scarabaeidae). Implications agronomiques". En Revue d'ecologie et de Biologie du Sol, (25:4), 413-434.

Stevenson, B. G. y Dindal, D. L. (1987). "Insect effects on decomposition of cow dung in microcosms". En Pedobiologia, (30), 81-92.

Singh, Y., Singh, B., Maskina, M. S. y Meelu, O.P. (1995). "Response of wet land rice to nitrogen from cattle manure and urea in a rice wheat rotation". En Tropical Agriculture, (72), 91-96.

Vitosh, M. L., Davis, J. F. y Knezek, B. D. (1973). “Long term. Effects of manure, fertilizer and plow depth on chemical properties of soil and nutrient movement in a monoculture corn system". En Journal Environmental Quality, (2), 269-299.

Yokoyama, K., Kai, H., Koga, T. y Aibe, T. (1991). "Nitrogen mineralization and microbial populations in cow dung, dung balls and underlying soil affected by paracoprid dung beetles". En Soil Biology and Biochemistry, (23:7), 649653. 